\title{
A!
}

This is an electronic reprint of the original article.

This reprint may differ from the original in pagination and typographic detail.

Österberg, Monika; Peresin, Maria S.; Johansson, Leena-Sisko; Tammelin, Tekla

\section{Clean and reactive nanostructured cellulose surface}

\section{Published in:}

Cellulose

DOI:

10.1007/s10570-013-9920-8

Published: 01/06/2013

Document Version

Peer reviewed version

Please cite the original version:

Österberg, M., Peresin, M. S., Johansson, L-S., \& Tammelin, T. (2013). Clean and reactive nanostructured cellulose surface. Cellulose, 20(3), 983-990. https://doi.org/10.1007/s10570-013-9920-8

This material is protected by copyright and other intellectual property rights, and duplication or sale of all or part of any of the repository collections is not permitted, except that material may be duplicated by you for your research use or educational purposes in electronic or print form. You must obtain permission for any other use. Electronic or print copies may not be offered, whether for sale or otherwise to anyone who is not an authorised user. 
1 Clean and reactive nanostructured cellulose surface 
Abstract:

A simple, solvent-free and low cost method to activate the surface of nanofibrillated cellulose

15 films for further functionalization is presented. The method is based on the oxidative properties of UV

16 radiation and ozone, to effectively remove contaminants from nanocellulosic surface, which remains

17 clean and reactive for at least a week. The efficiency of the method is demonstrated by X-ray

18 photoelectron spectroscopy and contact angle measurements. In clear contrast to previous results on

19 nanoscaled cellulose the relative atomic concentration of non-cellulosic carbon atoms was only $4 \%$, and

20 water completely wetted the surface within seconds. After activation, neither chemical degradation nor

21 morphological changes on cellulose were observed. This surface activation is essential for further

22 functionalization of the film in dry state or nonpolar media. The surface activation was confirmed by

23 silylation and a four times higher degree of substitution was achieved on the activated sample compared

24 to non-activated reference film, as monitored with XPS.

26 KEY WORDS: microfibrillated cellulose, ozonation, film, cleaning, UV

\section{Introduction}

29 Cellulose is a natural polymer and an almost unlimited source for sustainable production of materials on

30 industrial scale. Recently the interest for nano-scaled cellulosic materials, like nanofibrillated cellulose

31 (cellulose nanofibrils, NFC) or cellulose whiskers (cellulose nanocrystals, CNC) has increased

32 exponentially (Habibi et al. 2010, Klemm et al. 2011, Nishiyama et al. 2008, Walther et al. 2010). The

33 main reasons for the growing interest in nanocellulose are the development of energy efficient and up-

34 scalable production methods (Pääkkö et al. 2007, Saito et al. 2007, Wågberg et al. 2008) and the 
extraordinary properties of this renewable material (Eichhorn et al. 2010).

Due to the large surface area and enhanced hydrogen bonding ability of the fibrils, NFC forms strong hydrogels at very low solids content. Furthermore, upon drying the nanofibrillar network in these hydrogels has a high inherent tendency to form film-like structures which is especially interesting feature when considering novel application areas. NFC films are strong, they exhibit low oxygen permeability and they can appear as transparent or translucent plastic-like materials rendering them as 41 an attractive alternative to petroleum-based polymers for example in packaging applications (Lavoine et al. 2012). Moreover, we have developed relatively fast and up-scalable methods to prepare NFC films (Österberg et al. 2013, Peresin et al. 2012, Tammelin et al. 2011) which can be further surface modified e.g. in aqueous media (Orelma et al. 2012).

Nevertheless, the unique properties of NFC hydrogels change dramatically and often unpredictably upon drying or when exposed to nonpolar media. This is why most literature report on application or modification of NFC in never-dried form (Klemm et al. 2011). In our recent paper we proposed that this is due to the carbonaceous contamination layers that accumulates on the surface of highly hydrophilic cellulosic nanofibrils and nanofibrillar films whenever exposed to nonpolar media, e.g. upon drying (Johansson et al. 2011). The passivation layer impedes modification or application

51 based on interactions with the hydroxyl groups of cellulose, via occupying significant part of these groups so that they are no longer available for further functionalization. However, we also found that using appropriate solvent exchange procedure before drying of the NFC dispersion, the irreversible passivation of the surface was avoided and the surface of the individual nanofibrils were highly accessible for further functionalization (Johansson et al. 2011).

The remaining challenge is consequently how to re-activate dry nanocellulosic surfaces. In this contribution we introduce a solvent-free method, to clean and re-activate the NFC film surface, based on the combination of UV and ozone. Ozone is a powerful oxidation reagent used in many applications for 
removal of organic contaminants from various surfaces like steel (Kumar and Biswas 2011), sensor

60

61

62

1

ceramics (Chen et al. 2007, Zhang et al. 2006), and atomic force microscopy (AFM) probes (French et al. 2008). UV radiation in the presence of ozone $\left(\mathrm{UV} / \mathrm{O}_{3}\right)$ was also found to be the most efficient cleaning method in recycling gold nanoparticle junction arrays (French et al. 2008). Patterned superhydrophilic arrays have also been obtained by $\mathrm{UV} / \mathrm{O}_{3}$ treatment (Luz et al. 2011).

The effect of $\mathrm{UV} / \mathrm{O}_{3}$ on the surface properties of dry films of nanofibrillated cellulose was evaluated with atomic force microscopy (AFM), X-ray photoelectron spectroscopy (XPS), contact angle (CA) and FT-IR experiments. The enhanced reactivity and accessibility of the hydroxyl groups on the NFC film surface was verified with a simple and well-known silylation reaction. Significant improvement in the surface reactivity of NFC films was observed upon $\mathrm{UV} / \mathrm{O}_{3}$ activation without any severe changes in nanocellulose structure and chemistry.

\section{Experimental Section}

Nanofibrillated cellulose was prepared by disintegration of fully bleached never dried hard wood kraft pulp using a high shear Fluidizer (Microfluidics Int. Co, USA). The pulp was washed into sodium form prior to disintegration according to a method introduced by Swerin et al (1990) and passed six times through the fluidizer. This treatment removes excess of salt from the pulp suspension and ensures that only sodium ions are associated with anionic groups on the pulp. No chemical or enzymatic pretreatment was applied and the charge density of the pulp was $0.0065 \mathrm{meq} / \mathrm{g}$ as determined by conductometric titration according to Katz et al (1984). The $132 \mathrm{~cm}^{2}$ large and around $100 \mu \mathrm{m}$ thick free-standing NFC films were prepared by over-pressure filtration of a $0.84 \%$ dispersion of fibrils followed by hot pressing at $100^{\circ} \mathrm{C}$ and $1800 \mathrm{~Pa}$ (Österberg et al. 2013). Sefar Nitex polyamine fabric was used for filtration and remained with the film during pressing. A commercial UV ozonator from Bioforce Nanosciences was used to activate the NFC film surface. The nominal power of the UV source 
was $80 \mathrm{~W}$, the intensities of the most important wavelengths were 199 for $185 \mathrm{~nm}$ and 3975 for $254 \mathrm{~nm}$ according to the manufacturer. The distance between sample and radiation source was $2.5 \mathrm{~cm}$. Treatment time for the films was $10 \mathrm{~min}$. The sample size was about $2 \times 4 \mathrm{~cm}$.

Efficiency of the activation of the film surface was demonstrated by a simple silylation reaction performed on films obtained in pilot scale, according to Tammelin et al (2011) and Peresin et al (2012). The modification protocol is a slight variation of the method used to modify nanofibrils in bulk (Johansson et al. 2011). Films of about $100 \mathrm{mg}$ were submerged in $150 \mathrm{ml}$ Dimethylacetamide (DMA, $99 \%$ - Sigma-Aldrich) in a reaction vessel under continuous Nitrogen flow to evacuate any remaining oxygen. The film was kept in DMA under magnetic agitation until the temperature reached $80{ }^{\circ} \mathrm{C}$. Hexamethyldisilazan, (>99\% purchased from Sigma-Aldrich) was added drop-wise while stirring (1 $\mu 1$ reagent/mg of film). Reaction was continued over night at constant temperature. Films were thoroughly washed with DMA and with methanol (HPLC grade; Rathburn) to remove any possible unreacted chemicals. Finally, the films were allowed to dry in air.

X-ray photoelectron spectroscopy, XPS (Kratos Analytical AXIS 165 electron spectrometer with monochromatic A1 $\mathrm{K} \alpha$ irradiation at $100 \mathrm{~W}$ ), was used for surface chemical analysis of the freestanding NFC films. More specifically the effect of $\mathrm{UV} / \mathrm{O}_{3}$ treatment and silylation on surface chemical composition was monitored. The measurement procedures and acquisition parameters were optimized for cellulosic samples as described by Johansson (Johansson 2002). Samples were analysed after one week storage in desiccator. A minimum of three different areas were measured per sample.

AFM (Nanoscope IIIa scanning probe instrument from Digital Instruments, Santa Barbara, USA) was used to probe the surface topography of the films before and after $\mathrm{UV} / \mathrm{O}_{3}$ treatment. Three different areas on each sample were scanned in tapping mode in air using silicon cantilevers (SCD 15/AIBS, MicroMasch, Talinn, Estonia). The nominal resonance frequency of the cantilevers was around 300 $\mathrm{kHz}$. 
108 contact angle with a video camera based fully computer-controlled contact angle meter (CAM 200 from 109 KSV Instruments Ltd., Finland). The measurements were performed at room temperature using 110 deionized water further purified through a Milli-Q water purification unit. At least five separate 111 measurements were made for each sample either 15 min. or 1 week after $\mathrm{UV} / \mathrm{O}_{3}$ treatment. The samples 112 were stored in a desiccator prior to measurements.

113 Fourier transform infrared spectroscopy (FTIR) was applied to verify that no chemical 114 degradation of the cellulose occur during the UV/ozone treatment. With the BIO-RAD UMA 500 115 spectrometer (USA) equipped with a universal attenuated total reflection (ATR) germanium crystal the 116 films could be analyzed directly without further sample preparation, the analysis depth being about one 117 micrometer. The spectral data was processed with GRAMS AI software (Thermo Scientific, Galactic 118 Industries Corporation, New Hampshire, USA. All spectra were baseline-corrected and further 119 normalized to have the same value at $1200 \mathrm{~cm}-1$. An average spectrum of three measurements is 120 reported. Results and Discussion UV ozone treatment was evaluated as a method to clean the surface of NFC films. The method is 124 based on the power of ozone to decompose small molecular weight organic substances while not 125 affecting the cellulosic structure. UV light generates free radicals on the surface of low molecular 126 weight polymers leading to oxidative degradation. UV radiation $(185 \mathrm{~nm})$ reacts with atmospheric 127 oxygen and forms atomic oxygen and ozone, both strong oxidizers. Ozone absorbs the $254 \mathrm{~nm}$ UV 128 radiation and dissociates into molecular oxygen and atomic oxygen (Zhang et al. 2006). UV light 129 excites ambient oxygen molecules from their ground spin-triplet state into a spin-singlet state thus 130 creating a more reactive environment in which the immediate cleavage of organic bonds like C-C, C-H 
131 and $\mathrm{O}-\mathrm{H}$ into volatile organics takes place (Ye et al. 2005). This procedure has been found to be more 132 efficient than UV radiation or ozone treatment separately.

133 NFC films were prepared by overpressure filtration of native unmodified NFC (Österberg et al. 134 2013). After hot pressing, a strong, dense and translucent film was obtained within a couple of hours 135 (Fig. 1a). The density of the film is about $1250 \mathrm{~kg} / \mathrm{m}^{3}$ (Österberg et al. 2013). XPS wide spectra were 136 recorded to confirm that the NFC film does not adsorb any foreign chemicals from the fabric during 137 pressing. Only carbon and oxygen signals were observed (Online resources 1). The film was then 138 treated with ozone created by UV light in a commercial UV ozonator, thus removing much of the 139 organic airborne contaminants from the surface.

140 Fig. 1 a) Free-standing translucent NFC film with the diameter of $13 \mathrm{~cm}$. In the inset an AFM 141 topography image of a $9 \mu \mathrm{m} 2$ area of the film surface is shown. b) XPS C 1s high resolution regions of 142 untreated film (blue lower spectrum) and $\mathrm{UV} / \mathrm{O}_{3}$ treated film (red upper spectrum). The measurements 143 were done one week after treatment. Observe the reduction in the $\mathrm{C}-\mathrm{C}$ carbon. c) Corresponding water 144 contact angle values for untreated reference (blue squares), $\mathrm{UV} / \mathrm{O}_{3}$ treated after $15 \mathrm{~min}$ (unfilled red 145 triangles) and after one week (red filled triangles). Note that due to the rapid spreading of the water 146 droplet only a few measurement points close to 0 s are visible $15 \mathrm{~min}$ after treatment. The surface topography of the film was homogeneous and with only nanometer scaled 148 roughness, as seen in the AFM image in the inset of Fig. 1a). In our previous work, we demonstrated 149 that although pure and reactive when dispersed in aqueous media, NFC easily accumulates substantial 150 amount of non-cellulosic material upon drying which passivates the fibril surfaces (Johansson et al. 151 2011). Experimental evidence of such behavior was obtained by X-ray photoelectron spectroscopy 152 (XPS). A high amount of carbon atoms which are bonded only to other carbon or hydrogen atoms (C-C 153 carbon signal) was observed at the outmost surface of the nanofibrillated cellulose. Similar behaviour 154 was observed in the case of untreated film surface (Fig. 1b). The relative abundance of C-C carbon was 
approximately $11 \%$ for the untreated nanocellulose films which is comparable to the previously reported results for nanocellulosic fibrils (Andresen et al. 2007, Littunen et al. 2011, Uschanov et al. 2011). Similar trend was perceived in the case of water contact angle values (Fig. 1c). The contact angle for untreated film was around $30^{\circ}$ in correlation with our previous findings, indicating the presence of a passivation layer on the film surface. Nevertheless, after treating the sample with $\mathrm{UV} / \mathrm{O}_{3}$ for ten minutes, the hydrophilicity of the sample increased and the water droplet completely wetted the surface within a few seconds. Simultaneously the C-C carbon signal decreased to $4 \%$, a very low value for nano-scaled cellulose samples and a $36 \%$ decrease as compared to untreated sample.

It is worth noting that the surface remained very hydrophilic for at least one week after the $\mathrm{UV} / \mathrm{O}_{3}$ treatment, as evidenced by contact angle values (Fig. 1c), suggesting that passivation of this surface after $\mathrm{UV} / \mathrm{O}_{3}$ treatment is very slow. This behavior is the opposite to the one observed for most mineral surfaces. After the same cleaning procedure, silica surfaces are extremely reactive. However, they accumulate airborne contaminants within minutes when kept in ambient air. We suggest that the unique behavior observed for cellulosic substrates is due to the amphiphilic character of the cellulose molecule and its ability to rearrange the outermost surface molecules, hence minimizing its surface free energy. This behavior may well be one reason for the overall stability of cellulose materials (Johansson et al. 2011, Johansson et al. 2012). The rearrangement at the surface is reversible and thus, the cellulose is fully reactive when immersed in aqueous media where the hydrophilic plane of the cellulose molecules adapts to the hydrophilic media. It must be noted that although the NFC film was made from nanofibrils, after the film formation and drying, it can be compared to macroscopic cellulose samples like filter paper or wood fibres. During the drying process, hydrogen bonds are formed between fibrils and although there is no evidence of increase in crystallinity, during the pressing the films become very dense (Österberg et al. 2013). If, on the other hand, the same treatment is conducted on nano-scaled ultrathin films of dispersed NFC fibrils, the same hydrophilicity and cleanliness is achieved but the film is considerably more reactive and accumulates a passivation layer over time (results not shown). The 
ultrathin film is more porous and thus has higher surface area, thus being less stable than macroscopic

181 cellulose films.

The effect of $\mathrm{UV} / \mathrm{O}_{3}$ treatment on activation of the surface was corroborated by means of a simple surface reaction. Chemical modification through silylation chemistry was performed directly on the surface of the dense NFC film before and after exposing the film surface to the $\mathrm{UV} / \mathrm{O}_{3}$ treatment. The results of the reaction were evaluated by XPS analysis. XPS high resolution spectra for carbon (C1s) and silicon (Si2p) are shown in Fig. 2.

Fig. 2 XPS C 1s and Si 2p high resolution regions of untreated film (blue bottom spectrum), untreated and silylated film (black middle spectrum) and $\mathrm{UV} / \mathrm{O}_{3}$ activated and silylated film (red upper spectrum).

In the high resolution carbon 1s spectra no clear differences between unmodified (blue) and

191 silylated unactivated film is observed. However an increase in the peak around $284 \mathrm{eV}$ is observed for 192 the activated and silylated sample. In this region both $\mathrm{C}-\mathrm{C}$ bonds and C-Si bonds appear. In the high 193 resolution Si $2 p$ spectra the effect of surface activation is clearly seen. A small increase in the Si band is 194 observed for the silylated unmodified sample (black spectrum) but the increase is much higher for the 195 activated and silylated sample (red spectrum). The degree of substitution was calculated based on the 196 relative amount of silicon on the surface and was 0.07 for un-activated and 0.26 for $\mathrm{UV} / \mathrm{O}_{3}$-activated 197 sample, corresponding to a four-fold increase in surface activity due to the $\mathrm{UV} / \mathrm{O}_{3}$ treatment. From the 198 wide spectra and high resolution oxygen 1s spectra for the respective samples (Online resource 2 in 199 electronic online resources) we conclude that no significant chemical change except the silylation 200 reaction is occurring at the surface.

201 The oxidative reaction induced by ozone is very strong, introducing carboxylic acid or carbonyl 202 groups at the surface, and leading to severe degradation of low molecular weight polymeric substrates 203 like rubber or low density polyethene (Hedenberg and Gatenholm 1996, Romero-Sánchez et al. 2005), 
There is no available literature on the effect of ozone on NFC, although ozone is commonly used in 205 chlorine free delignification of pulp in the papermaking industry (Katz and Scallan 1983). Koljonen et 206 al. (Koljonen et al. 2003) demonstrated that the ozone oxidizes lignin on the pulp fibre surface, which is 207 seen as a decrease in $\mathrm{C}-\mathrm{C}$ carbon (present in lignin) and increase in the $\mathrm{O}-\mathrm{C}=\mathrm{O}$ carbon in XPS data. 208 Yet, AFM imaging revealed that the cellulose microfibrils stayed intact during the ozone treatment. 209 Ozonation has moreover been used to increase the adhesion between low density polyethene and 210 cellulose fibres in composites (Hedenberg and Gatenholm 1996, Kurosu et al. 2009). As expected, while 211 ozone treatment of polyethene was proven to be efficient ozone treatment of cellulosic fibres decreased 212 the overall adhesion. In photochemical bleaching of pulp (Ohtsuki et al. 2011) or wood powder (Kurosu 213 et al. 2009) the power of $\mathrm{UV} / \mathrm{O}_{3}$ treatment is utilized to remove lignin without decreasing the strength of 214 the pulp (Kurosu et al. 2009). To ensure that there was no degradation occurring at the NFC film surface 215 due to the oxidation, the films were imaged with AFM before and after treatment and the chemical 216 composition of the film was evaluated using attenuated total reflection (ATR) IR (Fig. 3).

217 Fig. 3 a) AFM topography images, and b) one representative line scan for untreated NFC free-standing 218 film (NFC reference, left) and NFC film that have been $\mathrm{UV} / \mathrm{O}_{3}$ treated (right). c) ATR IR spectra for the 219 two samples illustrate that the sample is not chemically modified due to the ozonation. The area of 220 dashed rectangle from $2000-1200 \mathrm{~cm}^{-1}$ is shown magnified in the inset. Since the spectra overlap the $221 \mathrm{UV} / \mathrm{O}_{3}$ spectrum was shifted up by 1 a.u. in the inset to facilitate comparison (the upper curve 222 corresponds to $\mathrm{UV} / \mathrm{O}_{3}$ treated sample).

From the AFM topography images (Fig. 3a) and the representative lines scans (Fig. 3b) it is obvious that the fibrillar structure was maintained and there is no evidence of changes in the fibril width that would indicate degradation although a decrease in molecular weight cannot be excluded based on 226 these results. The carbonyl groups are very IR active and are usually seen in ozone treated polymer 227 films in the $1700-1750 \mathrm{~cm}^{-1}$ region although the reaction is taking place at the surface (Hedenberg and 
Gatenholm 1996). However, as seen in the magnification of this region there are absolutely no changes

of the ATR IR spectra for the film before and after $\mathrm{UV} / \mathrm{O}_{3}$ treatment. As a matter of fact no chemical changes are observed in the spectra. We further note that the carbon peak in XPS spectra (Fig. 1b) is well resolved and there are no significant changes occurring in the $\mathrm{C} 4$ region suggesting that the surface retains its chemical structure. This is in contrast to e.g. oxygen plasma treatment of cellulose fibres, where quite short treatment times induces clear degradation, observed as increase in $\mathrm{C}-\mathrm{C}$ carbon and oxidation, observed as an increase in $\mathrm{O}-\mathrm{C}=\mathrm{O}$ carbon (Carlsson and Ström 1991). peculiar features: the strong self-association tendency of nanofibrils during drying creates smooth, strong, dense, flexible, transparent/translucent films with low transmission rates of gas molecules also at humid conditions (Österberg et al. 2013, Sehaqui et al. 2011). Recently it has been shown that the film manufacturing technology is up-scalable in order to produce films using industrially relevant roll-to-roll technique (Tammelin et al. 2011). For optimum performance and in order to create functional materials there is a need to modify the surface by chemical reactions. Therefore a simple, solvent-free cleaning method, such as the one presented here, should be highly beneficial to boost the film surface reactivity for further functionalizations, eliminating one of the obstacles hindering industrial applications of NFC.

A simple solvent-free method to clean and activate the NFC film surface was demonstrated. The method efficient functionalization. Almost four times higher degree of silylation was achieved on activated NFC

films. Additionally, the effect was observed to be long-lasting and the treatment did not degrade the cellulose. In this respect cellulose differs from both ceramics and metals that stay clean for only minutes or other organic materials that are degraded by ozone. Due to the recent advances in up-scaling both 
253 NFC production and NFC film manufacturing this simple method, based on a commercial technique, to

254 tailor the surface of the NFC film may have large practical implication.

\section{Electronic Supplementary Material}

256

XPS Wide spectra of untreated NFC film and $\mathrm{UV} / \mathrm{O}_{3}$ treated films before (Online Resource 1) and after (Online Resource 2) silylation are shown in the Electronic Supplementary Material.

\section{Acknowledgements}

Finnish Funding Agency for Technology and Innovation, Tekes, and companies within the Naseva II project are acknowledged for financial support. The NFC sample was received from UPM Kymmene corporation. Dr Joseph Cambell is thanked for the XPS analysis and Ms Anu Anttila, Ritva Kivelä, Rita Hatakka and Mirja Reinikainen are thanked for their excellent laboratory assistance. M.Sc. Anni Lähdetie and Raili Pönni are thanked for advice regarding FTIR data analysis.

\section{References}

Andresen M, Stenstad P, Møretrø T, Langsrud S, Syverud K, Johansson L, Stenius P (2007) Nonleaching Antimicrobial Films Prepared from Surface-Modified Microfibrillated Cellulose. Biomacromolecules 8:2149-2155.

Carlsson CMG, Ström G (1991) Reduction and oxidation of cellulose surfaces by means of cold plasma. Langmuir 7:2492-2497.

Chen KL, Mylon SE, Elimelech M (2007) Enhanced aggregation of alginate-coated iron oxide (hematite) nanoparticles in the presence of calcium, strontium, and barium cations. Langmuir 23:59205928.

Eichhorn S, Dufresne A, Aranguren M, Marcovich N, Capadona J, Rowan S, Weder C, Thielemans W, Roman M, Renneckar S, Gindl W, Veigel S, Keckes J, Yano H, Abe K, Nogi M, Nakagaito A, Mangalam A, Simonsen J, Benight A, Bismarck A, Berglund L, Peijs T (2010) Review: current international research into cellulose nanofibres and nanocomposites. J. Mater. Sci. 45:1-33.

French RW, Milsom EV, Moskalenko AV, Gordeev SN, Marken F (2008) Assembly, conductivity, and 
chemical reactivity of sub-monolayer gold nanoparticle junction arrays. Sensors Actuators B: Chem. 129:947-952.

282

Habibi Y, Lucia LA, Rojas OJ (2010) Cellulose Nanocrystals: Chemistry, Self-Assembly, and Applications. Chem. Rev. 110:3479-3500.

Hedenberg P, Gatenholm P (1996) Conversion of plastic/cellulose waste into composites. II. Improving adhesion between polyethylene and cellulose using ozone. J Appl Polym Sci 60:2377-2385.

Johansson L, Campbell JM, Hänninen T, Ganne-Chèdeville C, Vuorinen T, Hughes M, Laine J (2012) XPS and the medium-dependent surface adaptation of cellulose in wood. Surf Interface Anal 44:899903.

Johansson L, Tammelin T, Campbell JM, Setälä H, Österberg M (2011) Experimental evidence on medium driven cellulose surface adaptation demonstrated using nanofibrillated cellulose. Soft Matter 7:10917-10924.

Johansson L (2002) Monitoring Fibre Surfaces with XPS in Papermaking Processes. Microchimica Acta 138:217-223.

Katz, S, Beatson RP, and Scallan, AM (1984) The determination of strong and weak acidic groups in sulfite pulp. Svensk Papperstidning, 87:R48-R53.

Katz S, Scallan AM (1983) Ozone and caustic soda treatments of mechanical pulp. Tappi J. 66:85-87.

Klemm D, Kramer F, Moritz S, Lindström T, Ankerfors M, Gray D, Dorris A (2011) Nanocelluloses: A New Family of Nature-Based Materials. Angew. Chem. Int. Ed. 50:5438-5466.

Koljonen K, Österberg M, Johansson L, Stenius P (2003) Surface chemistry and morphology of different mechanical pulps determined by ESCA and AFM. Colloids and Surfaces A: Physicochemical and Engineering Aspects 228:143-158.

Kumar D, Biswas SK (2011) Effect of surfactant dispersed in oil on interaction force between an oil film and a steel substrate in water. Colloids Surf A 377:195-204.

Kurosu K, Miyawaki S, Ochi T (2009) Photochemical bleaching of kraft pulp by UV radiation. Kami Pa Gikyoshi 63:317-324.

Lavoine N, Desloges I, Dufresne A, Bras J (2012) Microfibrillated cellulose - Its barrier properties and applications in cellulosic materials: A review. Carbohydr. Polym. 90:735-764.

Littunen K, Hippi U, Johansson L, Österberg M, Tammelin T, Laine J, Seppälä J (2011) Free radical graft copolymerization of nanofibrillated cellulose with acrylic monomers. Carbohydr Polym 84:10391047.

Luz GM, Leite ÁJ, Neto AI, Song W, Mano JF (2011) Wettable arrays onto superhydrophobic surfaces for bioactivity testing of inorganic nanoparticles. Mater Lett 65:296-299.

Nishiyama Y, Johnson GP, French AD, Forsyth VT, Langan P (2008) Neutron Crystallography, 
Molecular Dynamics, and Quantum Mechanics Studies of the Nature of Hydrogen Bonding in Cellulose $315 \quad \hat{I}^{2}$. Biomacromolecules 9:3133-3140.

316 Ohtsuki T, Noda S, Ui S (2011) Improvements of bioconversion suitability of Japanese cypress wood by combination of UV radiation, ozonation and decay treatment with white-rot and brown-rot fungi.

Pääkkö M, Ankerfors M, Kosonen H, Nykänen A, Ahola S, Österberg M, Ruokolainen J, Laine J, Larsson PT, Ikkala O, Lindström T (2007) Enzymatic Hydrolysis Combined with Mechanical Shearing and High-Pressure Homogenization for Nanoscale Cellulose Fibrils and Strong Gels. Biomacromolecules 8:1934-1941.

Peresin MS, Vartiainen J, Kunnari V, Kaljunen T, Tammelin T, Quintus P (2012) Large-scale nanofibrillated cellulose film: an overview on its production, properties, and potential applications Book of Abstracts International Conference of Pulping, Papermaking and Biotechnology ; 2012 .

Romero-Sánchez MD, Mercedes Pastor-Blas M, Martín-Martínez JM, Walzak MJ (2005) Addition of ozone in the UV radiation treatment of a synthetic styrene-butadiene-styrene (SBS) rubber. Int. J. Adhes. Adhes. 25:358-370.

Saito, T, Nishiyama, Y, Putaux, J.-L, Vignon, M, Isogai, A (2006) Biomacromolecules 7:1687-1691.

Sehaqui H, Zhou Q, Berglund LA (2011) Mechanical performance tailoring of tough ultra-high porosity foams prepared from cellulose I nanofiber suspensions. Soft Matter 7:7342-7350.

Swerin, A, Ödberg, L, Lindström, T (1990) Nordic Pulp and Paper Research Journal 4:188-196.

Tammelin T, Salminen A, Hippi U (2011) FI20116048.

Uschanov P, Johansson L, Maunu S, Laine J (2011) Heterogeneous modification of various celluloses with fatty acids. Cellulose 18:393-404.

Wågberg L, Decher G, Norgren M, Lindström T, Ankerfors M, Axnäs K (2008) The Build-Up of Polyelectrolyte Multilayers of Microfibrillated Cellulose and Cationic Polyelectrolytes. Langmuir 24:784-795.

Walther A, Bjurhager I, Malho J, Pere J, Ruokolainen J, Berglund LA, Ikkala O (2010) Large-Area, Lightweight and Thick Biomimetic Composites with Superior Material Properties via Fast, Economic, and Green Pathways. Nano Letters 10:2742-2748-

Ye T, McArthur EA, Borguet E (2005) Mechanism of UV photoreactivity of alkylsiloxane selfassembled monolayers. J. Phys. Chem. B 109:9927-9938. 
350 Zhang J, Ebbens S, Chen X, Jin Z, Luk S, Madden C, Patel N, Roberts CJ (2006) Determination of the 351 surface free energy of crystalline and amorphous lactose by atomic force microscopy adhesion 352 measurement. J. Pharm. Res. 23:401-407. 